HIFAN 1675

\title{
Simulations of plasma confinement in an antihydrogen trap
}

\author{
K. Gomberoff \\ Department of Physics, University of California at Berkeley, Berkeley, California 94720-7300, USA, \\ Lawrence Berkeley National Laboratory, Berkeley, California 94720, USA, and Physics Department, \\ Technion, Haifa 32000 , Israel \\ J. Fajans \\ Department of Physics, University of California at Berkeley, Berkeley, California 94720-7300, USA \\ and Lawrence Berkeley National Laboratory, Berkeley, California 94720, USA \\ A. Friedman, D. Grote, and J.-L. Vay \\ Heavy Ion Fusion Virtual National Laboratory, Lawrence Berkeley National Laboratory, Berkeley, \\ California 94720, USA \\ J. S. Wurtele \\ Department of Physics, University of California at Berkeley, Berkeley, California 94720-7300, USA \\ and Lawrence Berkeley National Laboratory, Berkeley, California 94720, USA
}

\section{October 2007}

Accelerator Fusion Research Division

Ernest Orlando Lawrence Berkeley National Laboratory

University of California

Berkeley, California 94720

This work was supported by the Director, Office of Science, Office of Fusion Energy Sciences, of the U.S. Department of Energy under Contract No. DE-AC02-05CH11231. 


\title{
Simulations of plasma confinement in an antihydrogen trap
}

\author{
K. Gomberoff \\ Department of Physics, University of California at Berkeley, Berkeley, California 94720-7300, USA, \\ Lawrence Berkeley National Laboratory, Berkeley, California 94720, USA, and Physics Department, \\ Technion, Haifa 32000, Israel \\ J. Fajans \\ Department of Physics, University of California at Berkeley, Berkeley, California 94720-7300, USA \\ and Lawrence Berkeley National Laboratory, Berkeley, California 94720, USA
}

A. Friedman, D. Grote, and J.-L. Vay

Heavy Ion Fusion Virtual National Laboratory, Lawrence Berkeley National Laboratory, Berkeley, California 94720, USA

J. S. Wurtele

Department of Physics, University of California at Berkeley, Berkeley, California 94720-7300, USA and Lawrence Berkeley National Laboratory, Berkeley, California 94720, USA

The three-dimensional particle-in-cell (3-D PIC) simulation code WARP is used to study positron confinement in antihydrogen traps. The magnetic geometry is close to that of a UC Berkeley experiment conducted, with electrons, as part of the ALPHA collaboration [W. Bertsche et al., AIP Conf. Proc. 796, 301 (2005)]. In order to trap antihydrogen atoms, multipole magnetic fields are added to a conventional Malmberg-Penning trap. These multipole fields must be strong enough to confine the antihydrogen, leading to multipole field strengths at the trap wall comparable to those of the axial magnetic field. Numerical simulations reported here confirm recent experimental measurements of reduced particle confinement when a quadrupole field is added to a Malmberg-Penning trap. It is shown that, for parameters relevant to various antihydrogen experiments, the use of an octupole field significantly reduces the positron losses seen with a quadrupole field. A unique method for obtaining a 3-D equilibrium of the positrons in the trap with a collisionless PIC code was developed especially for the study of the antihydrogen trap; however, it is of practical use for other traps as well.

\section{INTRODUCTION}

Recent experiments ${ }^{1,2}$ have created antihydrogen by injecting cold antiprotons into a positron plasma confined in a Malmberg-Penning ${ }^{3}$ trap. The positrons are trapped axially, between two ring electrodes, and radially, by a strong axial magnetic field. These electric and magnetic fields do not confine the charge-neutral antihydrogen. Currently, it is thought that the only practical method of confining diamagnetic antihydrogen atoms is accomplished within a magnetic field minimum. The simplest field geometry that creates a magnetic field minimum employs two magnetic mirrors and a quadrupole, ${ }^{4,5}$ the so-called Ioffe-Pritchard trap. ${ }^{6}$ Clearly, these additional fields must not degrade the confinement of the charged positrons and antiprotons from which the antihydrogen is synthesized. However, recent experiments ${ }^{7,8}$ have shown that plasma confinement is sharply degraded by the superposition of weak quadrupole fields onto low solenoidal fields $(0.004-0.15 \mathrm{~T})$. This effect was attributed to resonant particle transport. ${ }^{7}$ Nonetheless, a single-particle model suggested that if the resonances can be avoided, there would be no significant loss. ${ }^{9}$

The early experiments ${ }^{7,8}$ used weak fields, though at ratios of multipole-to-solenoidal fields strength appropriate for antihydrogen experimentation. It had been hoped that plasma losses would be tolerable in the strong solenoidal fields used in current antihydrogen experiments. However, it is likely that trapping antihydrogen requires large quadrupolar fields. Even with a quadrupolar field large enough to create a $1 \mathrm{~T}$ field increase between the trap center and the trap wall, the well depth will only be $\sim 0.7 \mathrm{~K}$ for ground state antihydrogen. Evidence suggests that antihydrogen is formed with a kinetic energy significantly higher than this; in this case, such shallow wells will trap only a small fraction of the antihydrogen. Further experiments conducted using large solenoidal and quadrupolar fields have confirmed that application of the quadrupole field degrades the charged particle. ${ }^{10}$ These experiments showed that, for a wide range of system parameters, the quadrupole leads to fast ballistic loss of particles along field lines that intersect the confining wall of the trap.

Quadrupole fields increase linearly with $r$; i.e., the distance from the trap axis. Consequently, the field from the quadrupole is significant, even near the axis, and the positron and antiproton plasmas currently in use are unavoidably subject to this field. Accordingly, it has been suggested ${ }^{11}$ that the quadrupole be replaced by a higher order multipole. The near-axis field of a higher order multipole will be significantly lower than the field of a quadrupole with the same 
strength at the wall. Thus, the positron plasma, centered onaxis, would be subject to much lower perturbing fields, while the antihydrogen would be confined, albeit at a larger radius than in quadrupole confinement, by the multipole. With this motivation, a trap in which the quadrupole is replaced by an octupole has been proposed and studied experimentally. ${ }^{12,13}$ The use of a higher multipolar field in order to prevent high losses was reported recently; it was shown that, indeed, a sextupole field ${ }^{14}$ provides plasma confinement for time scales consistent with the production of antihydrogen.

Numerical investigations of particle confinement in antihydrogen traps are not performed easily. As the magnetic and electrostatic self-fields are not axisymmetric, the simulations must be fully three-dimensional (3-D). Furthermore, the characteristic time scales are widely separated: the positron cyclotron period is of order $\sim 10-30 \mathrm{ps}$; the positron axial end-to-end bounce time is several microseconds; the positron plasma column $\mathbf{E} \times \mathbf{B}$ rotation time is several tens of microseconds; and the diffusive loss time scale is seconds or more. Nonetheless, we have performed useful studies of confinement properties, using the three-dimensional particle-in-cell (PIC) simulation WARP. ${ }^{15}$ The simulations were performed with magnetic field strengths close to those of an experiment ${ }^{10}$ conducted at UC Berkeley as part of the ALPHA $^{12,16,17}$ antihydrogen collaboration.

In these studies, we have found that there is a characteristic critical radius for the initial plasma column, simply determined by the field geometry, beyond which particles are rapidly lost. This critical radius depends only on the order of the multipole and on a geometrical and field factor $\left(B_{w} L\right) /\left(B_{0} R_{w}\right)$, where $R_{w}$ is the radius of the trap at the wall, $L$ is the length of the plasma ( $L$ depends on the plasma parameters and on the electrostatic configuration), $B_{w}$ is the value of the multipole field at the wall, and $B_{0}$ is the axial magnetic field strength. We find, as expected, that the critical radius, for parameters relevant to antihydrogen experiments is larger for an octupole trap when compared to a quadrupole configuration.

The WARP ${ }^{15}$ code includes external magnetic and electric field elements and was originally designed for heavy ion beam simulations. It incorporates a special mover that allows the use of a time step that is larger than the cyclotron period, while still allowing for proper calculation of the orbital motion. ${ }^{18}$ In strongly magnetized plasmas, typical of antihydrogen experiments, the motion is primarily determined by the guiding center dynamics. WARP simulations can be run interactively, allowing, for example, switching from a twodimensional to a three-dimensional field solver during a simulation run.

We have performed two types of simulations. In order to estimate the critical radius, it is sufficient to consider simulations in which the positrons are injected into the trap, followed by rapid ramping of the multipole. These injection simulations reproduce analytical estimates for the ballistic loss of positrons along field lines. Additional physics was included in simulations that start from a plasma that is initially trapped and in global thermal equilibrium. These simulations used multipole ramp times longer than the $\mathbf{E} \times \mathbf{B}$ rotation period. The critical radius is close to that obtained with injected plasmas, but the details of the plasma distribution were somewhat different in the equilibrium simulations.

These simulations are the first 3-D PIC simulations that simulate a non-neutral plasma equilibrium in an antihydrogen trap. Furthermore, the method developed here, enables the study of the plasma properties in other trap configurations, either for the various antihydrogen experiments or for other non-neutral plasma traps.

Recent experimental results agree well with the fast ballistic process estimates that we show in our simulations. ${ }^{12,13}$ Further processes dictated by much slower time scales are more complicated to study. Although relatively large time steps have been used, the simulation is much too slow to mimic the experimental conditions precisely. In particular, practical multipole coils are ramped on over several seconds, a time much longer than it is feasible to model. In the experiment, the plasma temperatures are, typically, close to $4.2 \mathrm{~K}$, resulting in much shorter Debye lengths and much thinner plasma edges than what we can model in our simulations. Moreover, the simulation particles suffer illunderstood numerical collisions (i.e., there is a lack of knowledge of the effective characteristic parameters and operator of the collisions that depend on different nonphysical simulation parameters). Thus, for the purpose of studying realistically all types of transport processes that may occur, the numerical collisions are not useful since they do not properly model physical collisions. Furthermore, the numeric collisions require the use of a large number of macroparticles in order to avoid numerical heating.

However, the establishment of an initial local thermal equilibrium plasma makes use of the numerical collisions, as discussed in detail elsewhere. ${ }^{19,20}$ Thus, a new method has been developed ${ }^{19}$ that permits computing a positron equilibrium inside an antihydrogen trap or other non-neutral plasma traps. ${ }^{20}$

The paper is organized as follows. In Sec. II, trap geometry and magnetic field lines are discussed and an analytical expression for the critical radius is given. In Sec. III, we present a description of the injection and equilibrium simulations and of the corresponding trap parameters. We also outline the method for obtaining computational equilibria detailed elsewhere. ${ }^{19}$ In Sec. IV, we present results from injection simulations, and, in Sec. V, results are given for simulations with plasmas initially in thermal equilibrium. Note that the multipole ramp times used in Sec. V are longer than the $\mathbf{E} \times \mathbf{B}$ rotation time. A discussion is provided in Sec. VI.

\section{TRAP GEOMETRY AND MAGNETIC FIELD LINES}

A schematic of the Berkeley quadrupole experiment ${ }^{10}$ is shown in Fig. 1. Electrons injected from a negatively biased cathode (not shown in the schematic), entered the confinement region, and reflected from a negatively biased electrode at the opposite end of the trap. The confined plasma was created by a negative voltage applied to an electrode near the filament. The electrons were initially trapped with energies of a few electron volts, and subsequently cooled, via synchrotron emission, to the wall temperature $4 \mathrm{~K}$. At this point, 


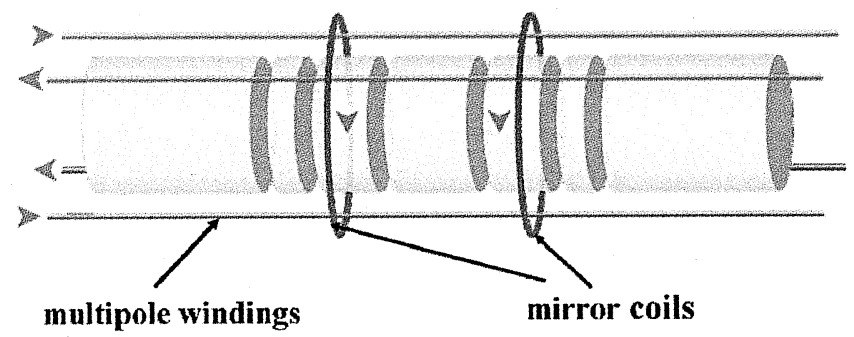

FIG. 1. (Color online) Schematics of an antihydrogen trap consisting of a Penning-Malmberg trap with multipole fields for radial confinement of antihydrogen. The mirror coils are shown for completeness, but were not included in the present simulations.

either a quadrupole or octupole coil was energized, and the plasma losses, if any, were observed.

The magnetic flux tube generated by a solenoid is a simple cylinder; multipole fields distort the field lines. For a quadrupole, the magnetic field is given by $\mathbf{B}=B_{w}\left(r / r_{w}\right)$ $\times[-\cos (2 \theta) \hat{\mathbf{r}}-\sin (2 \theta) \hat{\boldsymbol{\theta}}], \theta$ is the azimuthal angle, and the flux tube distorts such that a tube of circular cross section at the plasma center $(z=0)$ maps into ever more elongated ellipses. The ellipses at negative $z$ are rotated by $\pi / 2$ with respect to the corresponding ellipses at positive $z$. In general, field lines move radially and azimuthally. However, two field lines, at $\theta= \pm \pi / 2$, move only radially outward with increasing $z$, following a curve given by

$$
r=r_{0} \exp (\alpha z)
$$

where $\alpha=B_{w} / B_{0} R_{w}$. Here, $z$ is the axial position and $r$ the radial position along this field line, which has an initial radius $r_{0}$ at $z=0$. Two other field lines, at $\theta=0, \pi$, move radially inwards, following

$$
r=r_{0} \exp (-\alpha z)
$$

Figure 2 shows these field lines: An octupole field is given by $\mathbf{B}=B_{w}\left(r / r_{w}\right)^{3}[-\cos (4 \theta) \hat{\mathbf{r}}-\sin (4 \theta) \hat{\boldsymbol{\theta}}]$, and its flux tube (which has a circular cross section in the center) transforms into a four-lobed cylinder. Purely radial field lines satisfy

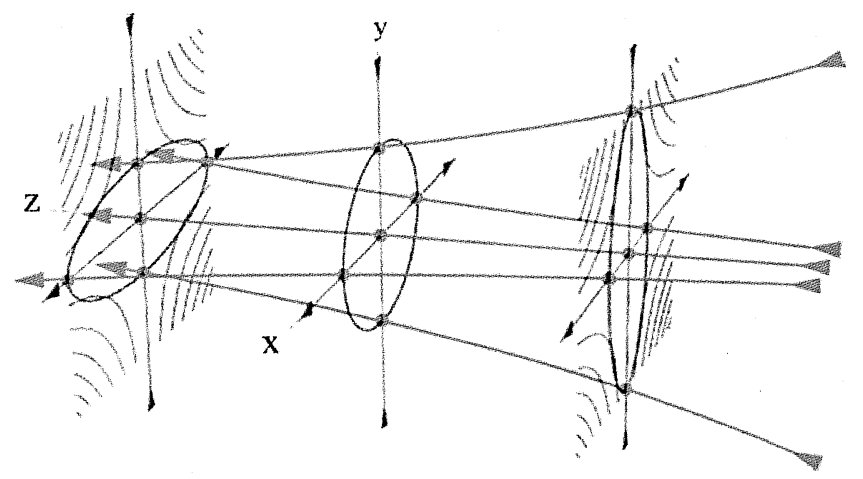

FIG. 2. (Color online) Magnetic field lines in a combined quadrupole and solenoidal field, and schematic plasma cross sections in three axial planes.

$$
r=\frac{r_{0}}{\sqrt{1-2 \frac{\alpha r_{0}^{2} z}{R_{w}^{2}}}}
$$

For a quadrupole, the general expression for the radius, i.e., $r(z)$, and angle $\theta(z)$ of a field line that passes through $\left(r_{0}, \theta_{0}, z=0\right)$, is

$$
\begin{aligned}
& r(z)=r_{0} \sqrt{\frac{\left(\mathrm{e}^{2 \alpha z}+e^{-2 \alpha z}\right)}{2}-\cos \left(2 \theta_{0}\right) \frac{\left(\mathrm{e}^{2 \alpha z}-e^{-2 \alpha z}\right)}{2}}, \\
& \theta(z)=\tan ^{-1}\left[\tan \left(\theta_{0}\right) e^{2 \alpha z}\right] .
\end{aligned}
$$

The field lines asymptote towards $\theta= \pm \pi / 2$ for positive $z$, and for negative $z$, towards $\theta=0, \pi$. At these angles, the field lines are radially directed.

The solenoidal fields used here are strong ( few Torr), such that the positrons follow guiding center orbits. Thus, positrons near $\theta= \pm \pi / 2$ and drifting towards positive $z$, will hit the trap wall if $r(+L / 2)$ exceeds the wall radius where $L$ was defined previously. Likewise, positrons near $\theta=0, \pi$ and drifting towards negative $z$ will hit the trap wall if $r(-L / 2)$ exceeds the wall radius. We define the critical radius $r_{c}(\theta)$ as the radial position of a positron initially at $z=0$, and at an angular position $\theta$ such that its trajectory intersects the trap wall at either $z=+L / 2$ or at $z=-L / 2 ; r_{c}(\theta)$ can be found by solving Eq. (4) for $r_{0}$.

Since the critical radius depends on the angle, the shape of the plasma in the center of the trap will no longer be circular. The critical radius $r_{c}(\theta)$, will be smallest for $\theta_{\min }$ $=0, \pi / 2, \pi$, and $3 \pi / 2$, and largest for $\theta_{\max }=\pi / 4,3 \pi / 4$, $5 \pi / 4$, and $7 \pi / 4$. At large $L$, the ratio between the largest to the smallest $r_{c}$ is $\sqrt{2}$. This is the same ratio as that of the diagonal to the side of a square; indeed, the shape of long plasmas subject to a quadrupole will approximate a square with corners at $\pi / 4,3 \pi / 4,5 \pi / 4$, and $7 \pi / 4$. The sides of the square will be closest to the center at $0, \pi / 2, \pi$, and $3 \pi / 2$, where the multipole field lines are purely radial. The lower bound for the average critical radius $r_{c}$ is given by $r_{c q}\left(\theta_{\min }\right)$, which can be written as

$$
r_{c q}=R_{w} \exp \left(-\frac{\alpha L}{2}\right) .
$$

This formula results from substituting $z=L / 2$ into Eq. (1). The corners of the center square remain sharp only if the positrons cannot cross field lines. In reality, $\mathbf{E} \times \mathbf{B}$ drifts from the plasma self-field and from the axial confinement fields cause the positrons to rotate around the trap axis. Even if a positron is on one of the corner $\theta_{\max }$ field lines, it will soon drift onto the more severely clipped field lines at $\theta_{\min }$. Simulations confirm that this rotation smooths the corners of the square. Thus, Eq. (5) gives an excellent overall estimate of $r_{c q}$. A similar argument yields

$$
r_{c o}=\frac{R_{w}}{\sqrt{(\alpha L+1)}}
$$

for the critical radius in an octupole field. 
Equations (5) and (6) illustrate an advantage of octupole field traps, as compared to quadrupolar field traps. Because $B_{w}$ and $B_{0}$ add in quadruture, $B_{w}$ must exceed $B_{0}$ to make a deep well. Moreover, electrostatics constraints make it difficult to trap a plasma that is shorter than the wall radius $R_{w}$. Thus, the scaling parameter $\alpha L$ will necessarily be close to unity, and perhaps be substantially greater than it. The quadrupole critical radius becomes exponentially small as $\alpha L$ increases and the plasma must have a very small radius, if it is to survive. In contrast, the octupole critical radius decreases quite slowly with $\alpha L$ and is always larger than the quadrupole critical radius.

\section{DESCRIPTION OF THE SIMULATIONS}

We performed two types of WARP simulations: injection and equilibrium simulations.

\section{A. Injection simulations}

In these simulations, the left-most electrode was grounded, allowing positrons to stream into the trap from the cathode. After the density in the trap stabilized, the potential on a grounded electrode near the cathode was raised to create an electrostatic well and trap most of the positrons. The positrons phase-mix in longitudinal phase space as they move in the external potential and self-fields; however, circulating electrostatic structures may remain. ${ }^{21,22}$ The multipole is not turned on until the simulations show significant phase mixing. The initial distribution thereby obtained is neither a global nor a local thermal equilibrium; the numeric collisions do not adequately mix particles across radial field lines. The plasmas are not even in local thermal equilibrium (LTE) along field lines; there is an overabundance of superthermal particles. (LTE here means that the particle velocity distribution has no net axial motion, is Maxwellian along field lines, and the density distribution obeys the Boltzmann relation in the self-consistent electrostatic field.) However, as discussed later, simulating plasmas initially in equilibrium is computationally more challenging, and the injection simulations provide a faster method for obtaining some of the important physical quantities of the system (e.g., $r_{c}$ ).

\section{B. Equilibrium simulations}

While more computationally expensive, equilibrium simulations are more realistic. We have developed a new and unique method for obtaining a 3-D computational equilibrium of non-neutral plasma inside a trap especially for the antihydrogen study. The numerical techniques used to obtain the proper numerical equilibrium, which are in local thermal equilibrium (LTE) along the field lines, are discussed in detail in a separate paper ${ }^{19}$ and used elsewhere to study thermal equilibrium in non-neutral plasma traps with magnetic mirrors. ${ }^{20}$

We here outline briefly the method. The new procedure follows a multistage approach. It requires a priori knowledge of the equilibrium of the trap in the absence of the slowly ramped multipole field elements. Starting from an idealized approximation of the equilibrium configuration in a coarse representation at lower dimensionality, the numerical parameters are slowly (almost adiabatically) modified toward a detailed solution of the full three-dimensional configuration. We use large-weight macroparticles and large time steps in the initial stages in order to obtain both, high numerical noise and rapid computational relaxation toward temporary and approximate equilibria. With this computational equilibrium, we can proceed to find the unknown equilibria in the presence of external fields by slowly ramping them.

In the antihydrogen trap study in order to initialize the plasma in the WARP code, an appropriate LTE density distribution was solved for with a code that solves the Boltzmann equation and Poisson equations self-consistently. ${ }^{23}$ Particles were loaded into the trap according to this LTE distribution, with a Gaussian velocity distribution function centered about $v=0$, and with the appropriate temperature.

Both simulations use similar electrode structures: the grounded electrode wall radius is $R_{w}=2.3 \mathrm{~cm}$, the central electrode is $7.0 \mathrm{~cm}$ long, and the end-electrode lengths are $2.0 \mathrm{~cm}$ for the injection simulations and $3.0 \mathrm{~cm}$ for equilibrium simulations. The plasma length is approximately $6 \mathrm{~cm}$; the exact length depends on the particle velocities, the radial positions, and the electrode voltages. Both simulations use the same solenoidal field, namely, $B_{0}=1 \mathrm{~T}$, as well as the same multipole field strength at the electrode wall: $B_{w} / B_{0}$ $=1.7$. Both types of simulations yield similar results. Further details concerning the simulations are provided in their respective sections.

\section{INJECTION SIMULATIONS}

In the injection simulations, positrons are emitted from a source biased to $5 \mathrm{~V}$; and they acquire velocities in the trap of about $10^{8} \mathrm{~cm} / \mathrm{s}$. The number of simulation particles is in the range of $10^{5}-10^{6}$ (depending on the initial plasma column size); there are about $40-70$ real particles per macroparticle and several tens of particles per cell. Typical round-trip times are on the order of $0.15-0.2 \mu \mathrm{s}$. The grid spacing in all dimensions (unless stated differently) is $0.1 \mathrm{~cm}$. The time step is $\Delta t=10^{-10} \mathrm{~s}$, or about three gyration periods. The plasma density is $\approx 10^{6} 1 / \mathrm{cm}^{3}$ and several initial radii of the plasma column are considered in the range of $r_{p}$ $=0.2-1.2 \mathrm{~cm}$.

Figure 3 shows a typical plasma evolution in a ramped quadrupole field. Plasma is continuously injected into the trap until, at $0.2 \mu \mathrm{s}$, the electrode near the injection is ramped, and the electrostatic well is fully established at $t$ $=0.4 \mu \mathrm{s}$. The plasma injection then ends, and only the trapped plasma remains in the simulation. Beginning at $t$ $=0.5 \mu \mathrm{s}$, a multipole (quadrupole or octupole) is ramped on over a period of $0.5 \mu \mathrm{s}$. At $1 \mu \mathrm{s}$, the multipole attains its final value of $B_{w}=1.7 \mathrm{~T}$ at the trap wall.

The plasma state is shown in Fig. 3 at for $t=0.6 \mu \mathrm{s}$ [(a)-(c)], $t=1 \mu \mathrm{s}[(\mathrm{d})-(\mathrm{f})]$, and $t=3 \mu \mathrm{s}[(\mathrm{g})-(\mathrm{i})]$. The effects of the quadrupole are evident. Initially, the plasma is circular near the axial center of the trap and becomes ellipsoidal at the axial edge. The ellipses at each end of the plasma are rotated by $\pi / 2$ with respect to each other, leading to the asymmetry visible in the $x-z$ space, as shown in Figs. 3(a), 

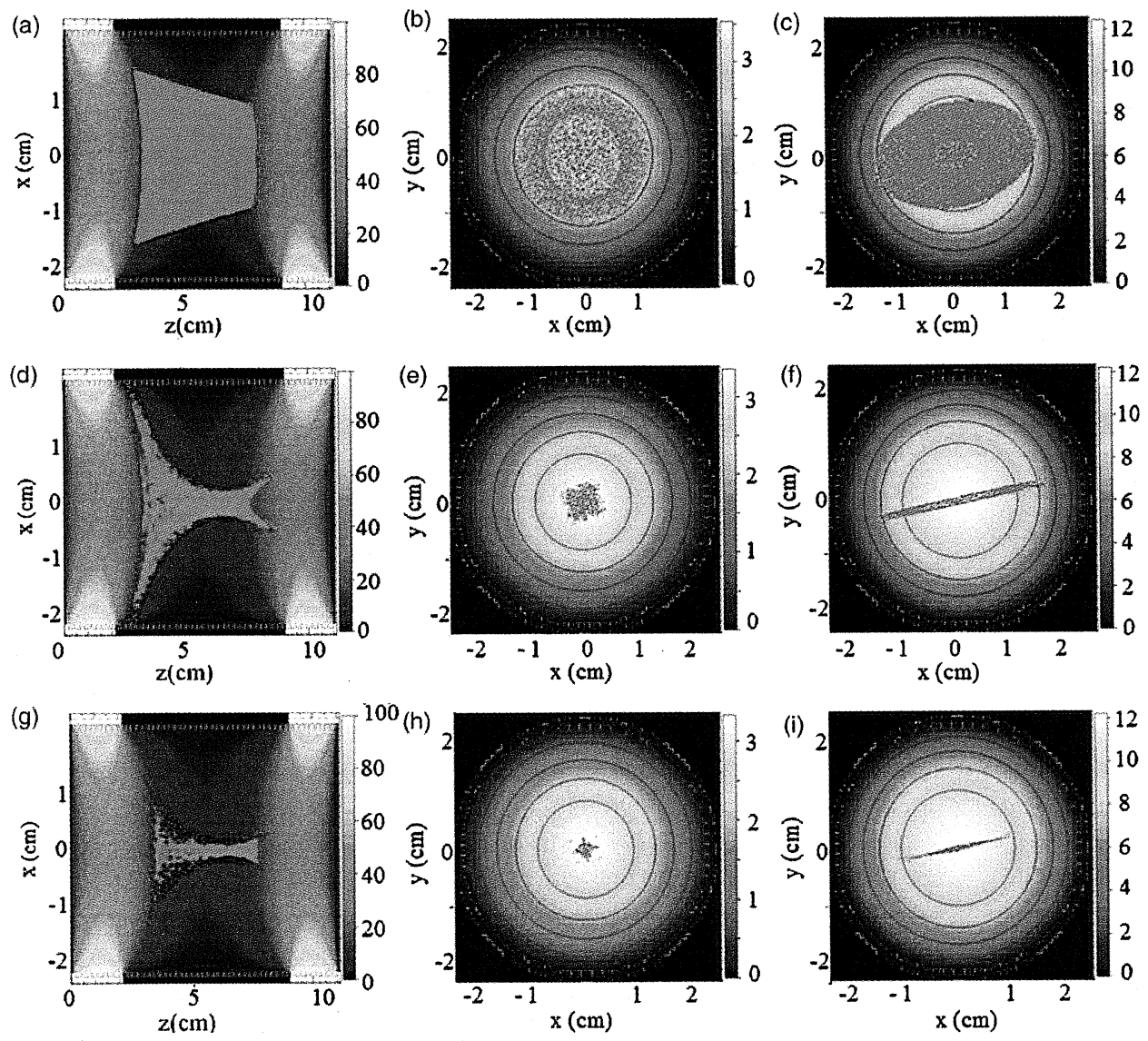

FIG. 3. (Color online) Quadrupole injection simulation results at $t=0.6 \mu \mathrm{s}$ [(a)-(c)] and $t=1 \mu \mathrm{s}[(\mathrm{d})-(\mathrm{f})]$, and $t$ $=3 \mu \mathrm{s}[(\mathrm{g})-(\mathrm{i})]$ for an initial plasma radius of $1.2 \mathrm{~cm}$. All plots have particles superimposed on a color map of the electrostatic potential. In (a), (d), and $(\mathrm{g})$, particles are plotted $z-x$ plane at $y=0$. That is, the potential is plotted at $y=0$ and all particles are projected along the $y$ coordinate. In (b), (e), and (h), the transverse $(x, y)$ position of particles in the center of the plasma (at the region $z=-0.1 \mathrm{~cm}$ to $z=0.1 \mathrm{~cm}$ ) are plotted with the potential at $z=0$. Plots (c), (f), and (i) are similar to (b), (e), and (h), but with the transverse cross section at one edge of the plasma $(z \approx 3 \mathrm{~cm})$. At $t=3 \mu \mathrm{s}$, most of the positrons have been lost. $3(\mathrm{~d})$, and $3(\mathrm{~g})$. Excluding the $\mathbf{E} \times \mathbf{B}$ rotation, as the quadrupole field is ramped, positrons attached to field lines that hit the confining wall are lost. This loss is responsible for the square shape of the plasma at $z=0$, as seen in Fig. 3(e). The square has diagonals that are rotated by $\pi / 4$ with respect to the ellipses at the edge (as predicted in the discussion of Sec. II). Further shape deformation occurs as remaining positrons undergo axial bouncing, and some $\mathbf{E} \times \mathbf{B}$ rotation; slow particles on undesirable field lines eventually leave the system. This is seen in Fig. 3(h), where the plasma has evolved to a diamondlike shape. Equation (4) predicts the elongated elliptical transverse shape of the plasma at its axial extremities [Figs. 3(c), 3(f), and 3(i)]. In Sec. V, it will be shown that ramping times that are slow compared to the $\mathbf{E} \times \mathbf{B}$ rotation time yield more rounded profiles at the plasma center.

At $t=3 \mu \mathrm{s}$, most of the positrons have been lost along field lines that intersect the confining wall. The final plasma size, i.e., $0.2-0.3 \mathrm{~cm}$, is of the order of the critical radius, as shown below.

As shown in Fig. 4, losses are dramatically lower for an octupole and the plasma radius is significantly larger, than for a quadrupole field. Figure 5 shows the advantage of octupole traps through a graph of the number of simulation particles as a function of time for three cases: an octupole with initial plasma radius of $r_{p}=1.2 \mathrm{~cm}$; an octupole with an initial plasma radius of $r_{p}=0.98 \mathrm{~cm}$; and a quadrupole with an initial plasma radius of $r_{p}=1.2 \mathrm{~cm}$. At first $(t<0.1 \mu \mathrm{s})$, the number of particles increases rapidly, as positrons are emitted from the source and fill the trap. The number then decreases, for $0.1<t<0.4 \mu \mathrm{s}$, by approximately $25 \%$, as the trap length is effectively shortened by application of the electrostatic barrier near the injection region. At $t=0.4 \mu \mathrm{s}$, the positron source is abruptly turned off, leading to a small downward jump in the positron number (these correspond to the few particles adjacent to the electrode, which are not trapped by the barrier). The multipole is ramped on beginning at $t=0.5 \mu \mathrm{s}$, and particles are lost quickly after the critical radius becomes smaller than the plasma radius (at about $t=0.65 \mu \mathrm{s}$ for the quadrupole and $t=0.8 \mu \mathrm{s}$ for the $r_{p}$ $=1.2 \mathrm{~cm}$ octupole). At $t=1 \mu \mathrm{s}$, the multipoles are fully energized, and the fast ballistic loss ceases. Measurement of the plasma radius at the plasma axial center, as well as calculation of the critical radius as indicated in the next paragraph, yields a critical radius of the octupole of roughly $1 \mathrm{~cm}$, while the quadrupole critical radius is approximately $0.2 \mathrm{~cm}$.

Particles continue to be lost, though, in both the injection and equilibrium simulations, at a much lower rate, after the multipole reaches its maximum at $1 \mu \mathrm{s}$. This is caused by two effects. A few particles start with such a low axial velocity that they simply take a long time to leave the trap. More importantly, some particles are lost as they drift from those field lines on which they do not experience large radial excursions onto field lines on which radial excursions may take them to the wall. Finally, numeric collisions cause slow cross-field transport from confined to unconfined trajectories.

The detailed treatment of slow multipole ramping in antihydrogen traps, while important, requires very long simulations as well the inclusion of collisions and cyclotron 

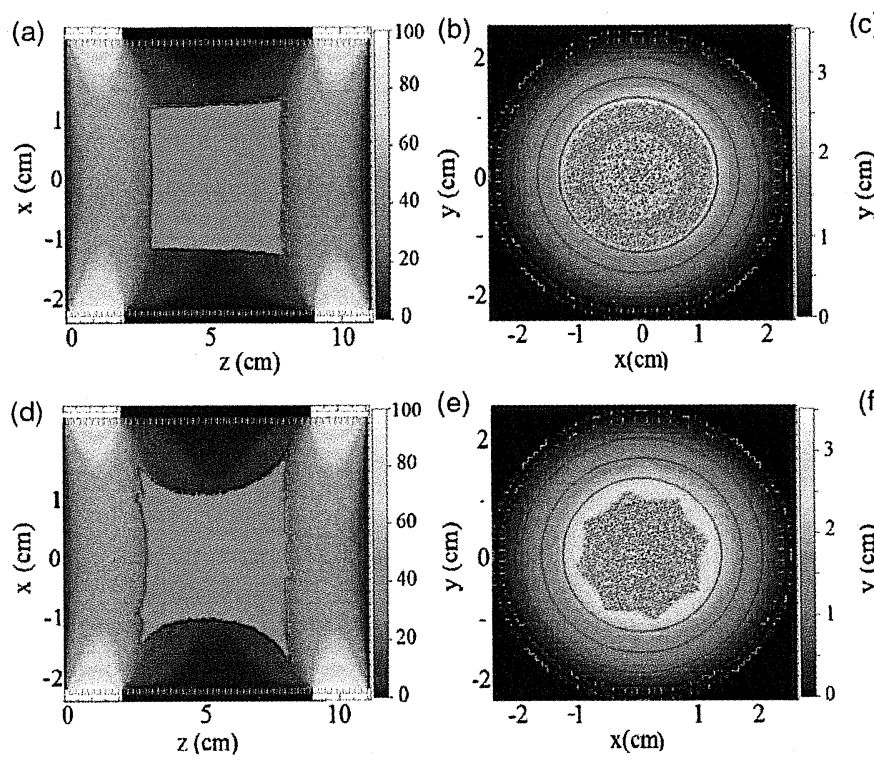
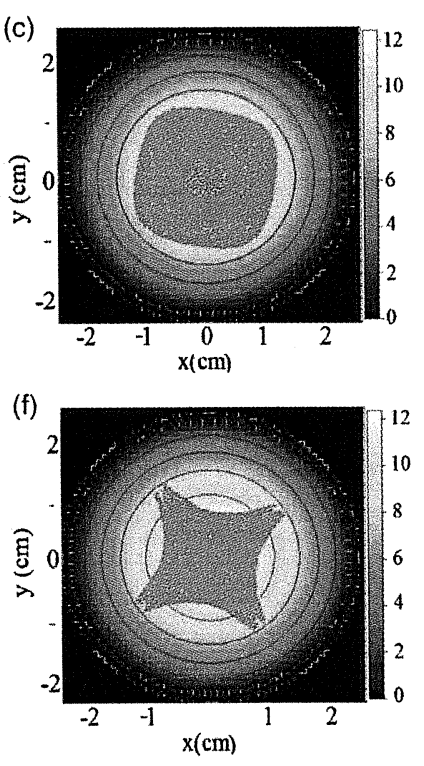

FIG. 4. (Color online) Octupole injection simulation results are plotted at $t$ $=0.6 \mu \mathrm{s}[(\mathrm{a})-(\mathrm{c})]$ and $t=3 \mu \mathrm{s}[(\mathrm{d})-(\mathrm{f})]$ for an initial plasma radius of $1.2 \mathrm{~cm}$. No substantial loss is evident. Subplots are the same as in Fig. 3 . damping. Study of these effects is outside the scope of the present paper.

From the initial number $\left(N_{i}\right)$ and final number $\left(N_{f}\right)$ of particles in the trap, we can calculate an effective "final" plasma radius: $\widetilde{r}_{f} \equiv r_{p} \sqrt{N_{f} / N_{i}}$. Roughly, a plasma that started with this final radius would not suffer ballistic loss after the application of the multipole field. If the critical radius is greater than the initial radius, $r_{p}>r_{c}$, then particles will be lost, and the final radius will equal the critical radius: $\widetilde{r}_{f}=r_{c}$. If, however, the initial radius is less than the critical radius, no particles will be lost, and $\tilde{r}_{f}=r_{p}$. Figure 6 shows $\widetilde{r}_{f}$ plotted as a function of the initial plasma radius for the quadrupole and octupole cases. As expected, the quadrupole final radius is pinned at about the critical radius of $0.20 \mathrm{~cm}$. The octu-

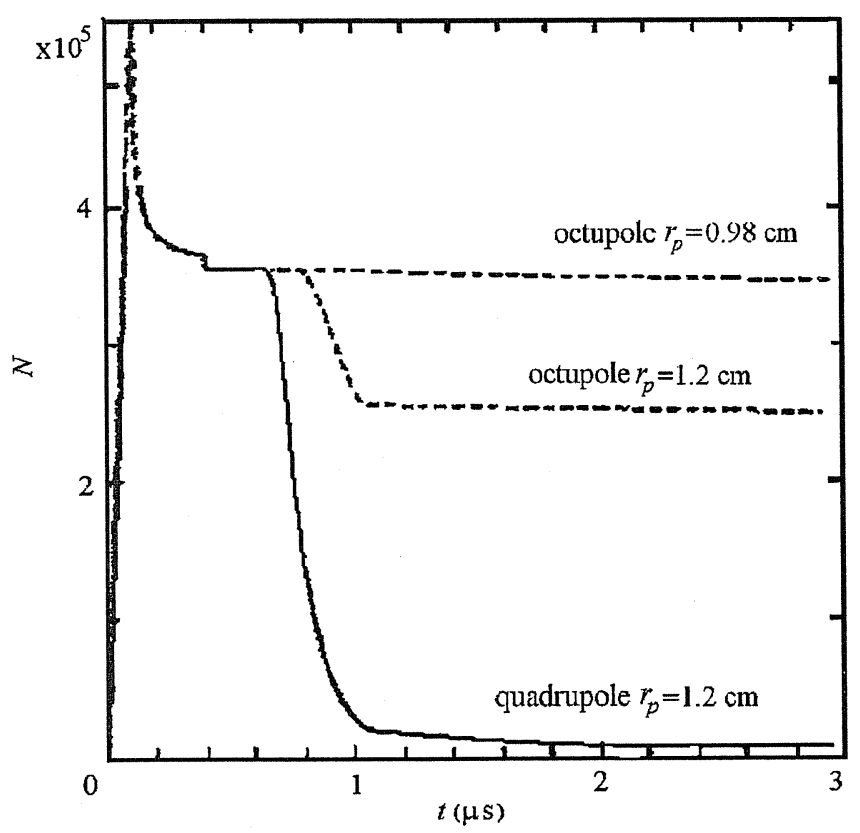

FIG. 5. Number of particles as a function of time for quadrupole and octupole cases. Different initial plasma radii of 1.2 and $0.98 \mathrm{~cm}$ are indicated. pole final radius equals the initial radius, until it exceeds the critical radius of about $1 \mathrm{~cm}$.

If we use a multipole ramping time that is substantially slower than the bounce time, the plasma particles will always be in rough equilibrium with the current value of the field as the field is ramped. By measuring the number of trapped particles, i.e., $N\left(B_{w}\right)$, as a function of the instantaneous multipole field $B_{w}$ at the wall, we can study the dependence of the critical radius on the multipole field strength. In Fig. 7, we show results for a simulation with a multipole ramp time of $3 \mu$ s (instead of $0.5 \mu$ s used earlier). We plot the critical radius as function of quadrupole and octupole field strength at the wall $\left[r_{f}\left(B_{w}\right)=r_{p} \sqrt{N\left(B_{w}\right) / N_{i}}\right]$. The simulation results confirm the analytical estimates of the critical radius. We find, for both the octupole and quadrupole cases, that the numerical results agree well with the analytical estimate, where $r_{c}$ is calculated, from Eqs. (5) and (6) at $z=L / 2$ $=2.9 \mathrm{~cm}$. The critical radius at $B_{w}=1.7 \mathrm{~T}$ is $0.99 \mathrm{~cm}$ for an octupole and $0.25 \mathrm{~cm}$ for a quadrupole (close to, but somewhat larger than the values obtained in the faster ramping

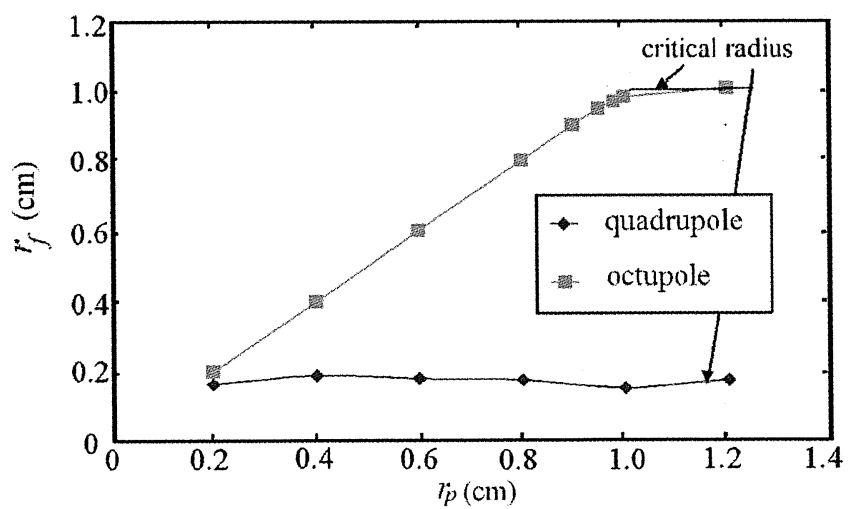

FIG. 6. (Color online) Final radius $\left[r_{f}=\sqrt{\left(N_{f} / N_{i}\right) r_{p}^{2}}\right]$ as a function of the initial radius. 

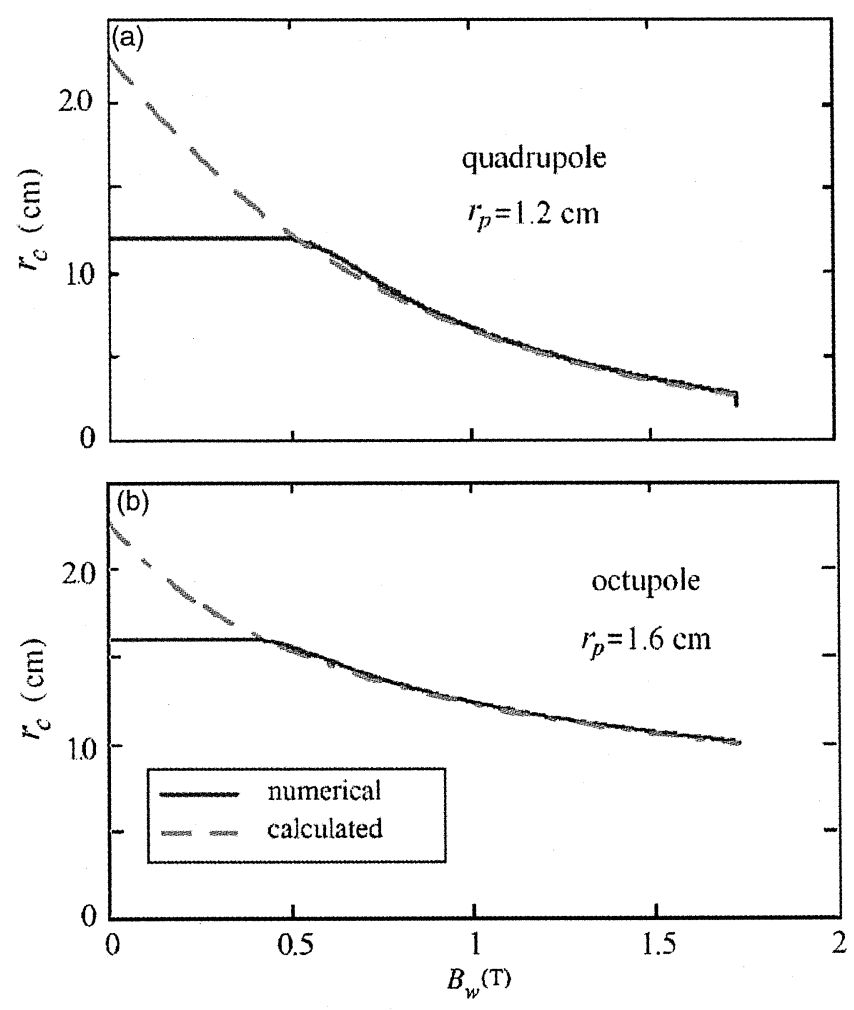

FIG. 7. (Color online) Numerical and analytical critical radius as a function of the magnetic field for (a) quadrupole and (b) octupole fields. In order to have more data for the octupole case, we start with a plasma with $r_{p}$ $=1.6 \mathrm{~cm}$ while, for the quadrupole, we start with $r_{p}=1.2 \mathrm{~cm} . r_{c}$ is calculated at $z=L / 2=2.9 \mathrm{~cm}$.

case). Thus, 16 times fewer particles remain in the trap with the quadrupole than with the octupole.

In addition to the injection runs presented here, we also considered injection simulations into a trap with pre-existing quadrupole or octupole fields. The critical radius can be calculated from the particle loss (after the potential wall of the trap has been established), and, for both quadrupole and octupole, they agree with the analytical expectations from Eqs. (1) and (3) (for a quadrupole and an octupole, respectively) when $z$ is replaced by $z=L$, rather than $z=L / 2$ [yielding equivalent expressions to those of Eqs. (5) and (6)]. In this case, the critical radius is smaller and the difference between the quadrupole and octupole case is even more pronounced.

\section{EQUILIBRIUM SIMULATIONS}

Due to superconducting technology limitations, multipole coils cannot be ramped on in less time than a few seconds. Since this time is much slower than the plasma collision time, it is expected that the plasma will remain in LTE during the ramping process. As noted earlier, our simulations are far too computationally intensive to run for this length of time. Nor can the simulations be run at plasma temperatures as low as typical for proposed antihydrogen experiments. At the experimental temperatures of $T \approx 0.5 \mathrm{meV}$, and at densities of $n \approx 2 \times 10^{7} \mathrm{~cm}^{-3}$, the Debye length is about $30 \mu \mathrm{m}$. The simulation would need a comparable grid size of at least 100 times finer resolution in the axial coordinate and would
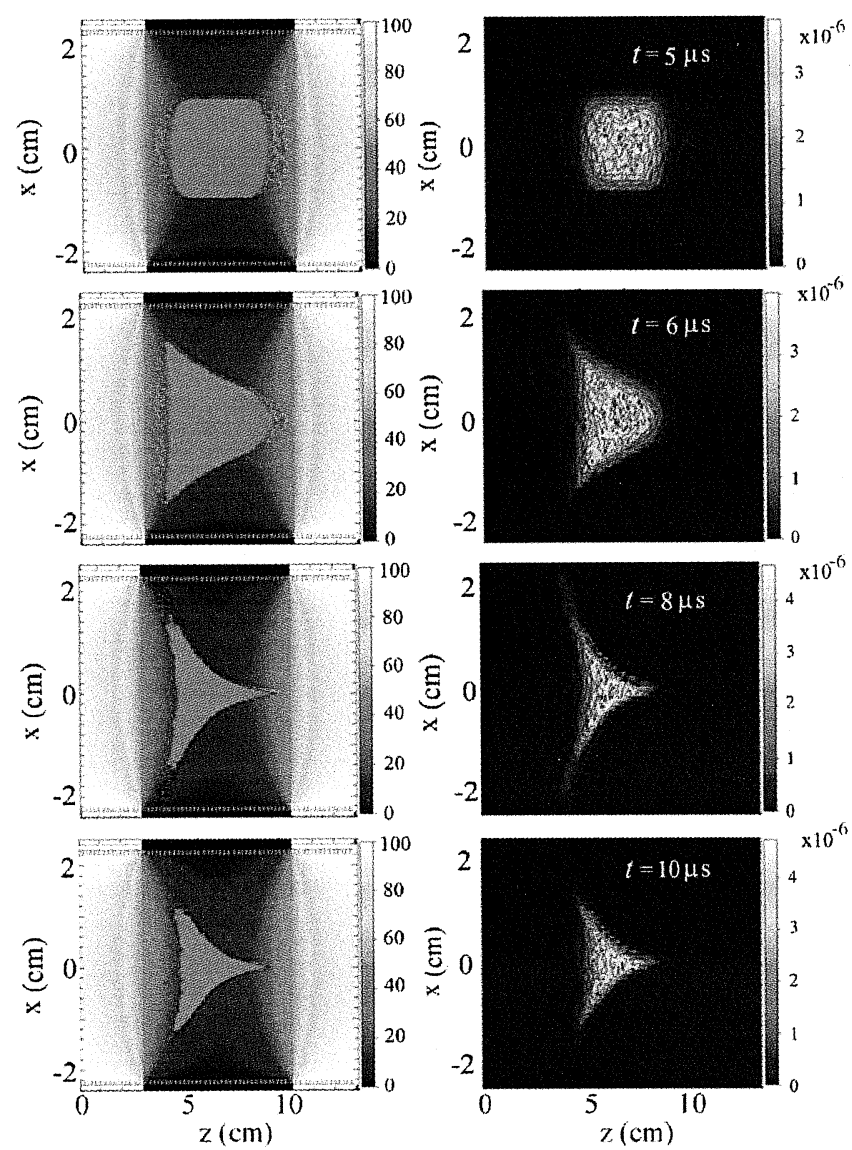

FIG. 8. (Color online) Equilibrium simulation results at times $t=5,6,8$, and $10 \mu \mathrm{s}$ for a quadrupole, with initial plasma radius $r_{p}=10 \mathrm{~mm}$ and a multipole ramp time of $3 \mu \mathrm{s}$. The plots on the left show the particle distribution, projected onto the $x-z$ plane, with the electrostatic potential superimposed. The charge density contours are shown on the right-hand side plots.

be prohibitively expensive. Consequently, we use positron temperatures of $T \approx 1 \mathrm{eV}$, and a maximum density of about $n \approx 2 \times 10^{7} \mathrm{~cm}^{-3}$.

The rapid loss of particles from an equilibrium plasma with a $1 \mathrm{~cm}$ initial radius is shown in Fig. 8. The quadrupole is turned on at $t=5 \mu \mathrm{s}$, and the ramping time of the quadrupole is $3 \mu \mathrm{s}$. At $t \sim 6.5 \mu \mathrm{s}$, particles begin to escape the trap. At $8 \mu \mathrm{s}$, the multipole field reaches its final value, and the density profile is strongly eroded. By $10 \mu \mathrm{s}$, the fast loss of particles has ceased. In Fig. 9, we plot the field lines and density contour (color map) when the quadrupole is at its final value. The plasma profile follows the field lines; however, this run is performed for a ramp time that is fast compared to $\mathbf{E} \times \mathbf{B}$ rotation, so particles do not cross the field lines.

We now describe simulations in which the multipole is ramped slowly, compared to the $\mathbf{E} \times \mathbf{B}$ rotation period. The simulations start from the same equilibrium $(T \approx 1 \mathrm{eV}, n$ $\approx 2 \times 10^{7} \mathrm{~cm}^{-3}$ ) as in the previous section. The multipole is turned on at $5 \mu \mathrm{s}$, and is ramped over $60 \mu \mathrm{s}$ (as opposed to $3 \mu \mathrm{s}$ ). For a flat-top plasma, the $\mathbf{E} \times \mathbf{B}$ rotation frequency can be estimated as $\omega=\frac{1}{2} \rho(r) / \varepsilon_{0} B$, leading to a period of $\sim 30 \mu \mathrm{s}$. However, the symmetry of the field lines repeats after a quarter of a rotation for a quadrupole, and repeats after an eighth of a rotation for an octupole. Therefore, the 


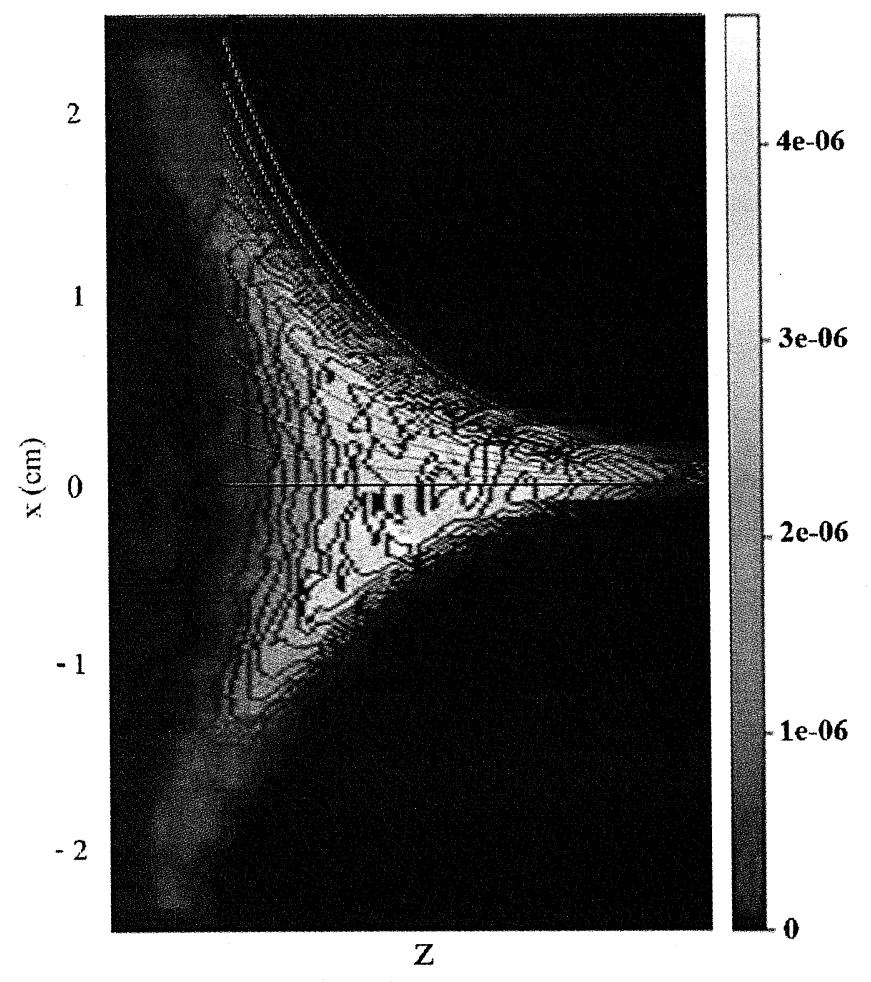

FIG. 9. (Color online) Quadrupole magnetic field lines and positron density color map with contour as seen when the multipole has reached its final value $(t=8 \mu \mathrm{s})$.

ramping of the multipole occurs over about 16 "effective" $\mathbf{E} \times \mathbf{B}$ rotation periods. (In the injection simulations, the density was about ten times lower and therefore the rotation time was about $300 \mu \mathrm{s}$.) We first consider the case of a quadrupole. After ramping the multipole, the simulation continues until $450 \mu \mathrm{s}$. In Fig. 10, we show the positron transverse distribution at times $t=65$ and $450 \mu \mathrm{s}$ (left and right plots, respectively). We see both a fast loss of particles, leading to Fig. 10(a), and a continuing loss, leading to the smaller distribution in Fig. 10(b). As the plasma in the equilibrium run further evolves, its shape becomes a rounded square, as expected, due the azimuthal drifts. Final dimensions of the plasma approach, approximately, those expected from the field line analysis, and the loss rate becomes small. For the quadrupole case, the longer ramp time does not improve the already poor plasma confinement.
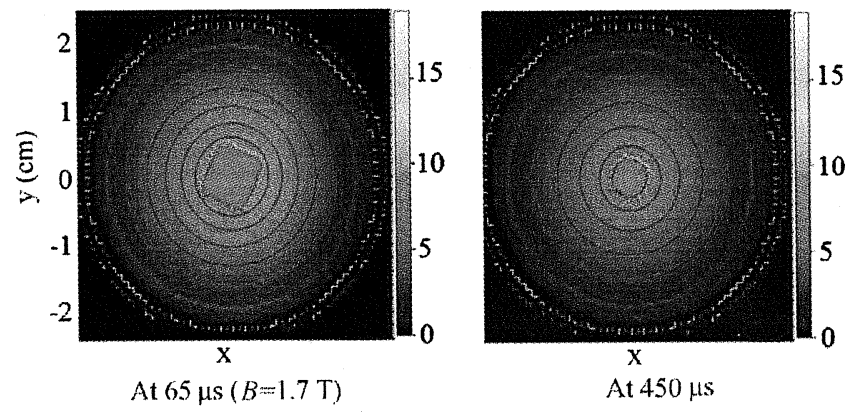

FIG. 10. (Color online) Positron phase space at the center of the plasma for two cases: Slow-ramping equilibrium simulation when the multipole field reaches its maximum $(t=65 \mu \mathrm{s})$, on the left; slow-ramping simulation at $t$ $=450 \mu \mathrm{s}$, on the right.

We now consider, for the same parameters, the octupole case. The results are shown in Fig. 11, where the transverse cross section is plotted at $t=220 \mu$ s [Fig. 11(a)], and a density contour plot is shown on the right [Fig. 11(b)]. The density plot was obtained by averaging the distribution over the $\sim 10^{3}(0.1 \mu \mathrm{s})$ time steps preceding $t=220 \mu \mathrm{s}$. The plasma is stable, with a radius of $1 \mathrm{~cm}$. While particles are lost in the corresponding injection runs (see Fig. 5) for an initial radius that is slightly larger than the critical radius, no particles are lost in the equilibrium run. This is due to the rotation drift, which is seen to increase the critical radius slightly.

\section{DISCUSSION}

We have performed the first 3-D PIC simulations of the confinement of the particles in an antihydrogen trap. These Malmberg-Penning traps have a superimposed multipole magnetic field so that they can simultaneously confine charged particles (positrons and antiprotons) and neutral particles (antihydrogen). The parameters used in the studies are close to those of a Berkeley experiment, which models the positron confinement in such a trap using electrons. The simulations show that confinement is degraded when a quadrupole field is employed. This is consistent with previous analytical estimates as well as with the experimental results. ${ }^{10}$ However, confinement in an octupole field is markedly improved. The primary loss mechanism on the time scales accessible with our code is due to the ballistic loss of
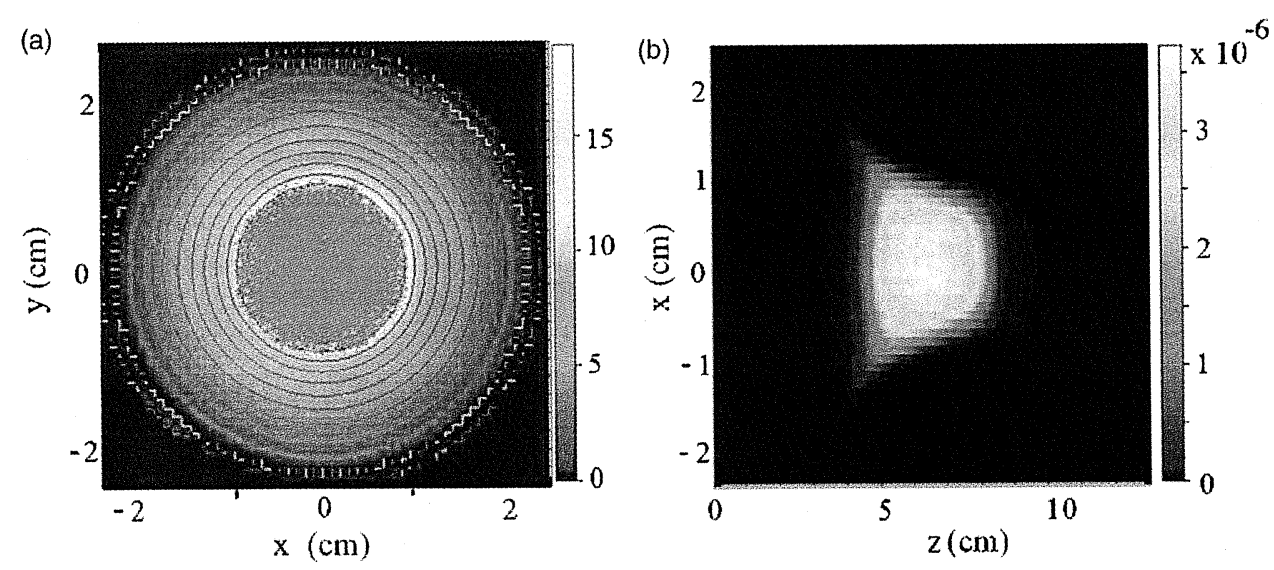

FIG. 11. (Color online) Simulation for an equilibrium simulation with a slowly ramped octupole and for $r_{p}$ $=1 \mathrm{~cm}$ at $t=220 \mu \mathrm{s}$. (a) Particle positions in $x-y$ space and the potential color map. (b) Color map of the density profile in the $x-z$ plane at $y=0$. 
particles along field lines that intersect the trap wall. The simulations confirm that there is a critical initial plasma radius below which ballistic loss does not occur. This critical radius found in the simulations agrees well with that estimated analytically. The critical radius depends directly on the order of the multipole and on a geometrical and field factor. It also depends on the effective length of the plasma which depends on the details of the electrostatic trap (for example, the shape of the external potential) and plasma parameters. For trap parameters used in the present work (appropriate to the ALPHA antihydrogen experiments), the number of trapped particles is larger by a factor of 16 . In fact, the recent experiments confirm the fast ballistic loss process and the superiority of the octupole field over a quadrupole field for the positron confinement, thus, being consistent with the present simulations. ${ }^{12,13}$ Furthermore, since the parameters used here are practical to many antihydrogen experiments, the results are relevant to these experiments as well. This suggests that the positron confinement will be improved by using a high multipolar magnetic field rather than a quadrupole in many experiments. Indeed, the use of a sextupole field has been already tested in an experiment with electrons motivated by antihydrogen trap experiments. ${ }^{14}$

The averaging from $\mathbf{E} \times \mathbf{B}$ rotation yields a more rounded profile, since particles cross the field lines as they rotate. We also find a small increase in the critical radius.

These simulations use plasma densities similar to those in the proposed experiments; however, computational limitations require warmer plasmas and faster ramping than planned for the experiments. The WARP particle mover properly calculates the various drifts (e.g., the $\mathbf{E} \times \mathbf{B}$ drift and the grad $\mathbf{B}$ drift) without resolving the cyclotron motion and, thus, without having to use correspondingly small time steps. Even so, equilibrium simulations with slow multipole ramping are relatively long.

Because of the computational limitations, and because WARP does not, at present, model real collisions or cyclotron cooling, we cannot use WARP to study the diffusive loss that we expect will dominate the plasma dynamics at long time scales. Future work may study these effects either with WARP, or a combination of WARP and codes that model the dynamics of test particles in the three-dimensional equilibria found by WARP. It is expected that the diffusive losses will be high for particles close to $r_{c}$, since these particles need only diffuse for a short distance to field lines that intersect the wall, and not across the longer distance to the wall found in the absence of the multipole field. Thus, $r_{c}$ likely sets an effective aperture for the plasma. Finally, the WARP simulations developed here are unique and first of their type and enable the study of the equilibria and evolution of nonneutral plasmas in traps with a collisionless PIC code. They can be used to simulate other trap configurations as well as a wide range of three-dimensional physics in non-neutral plas- mas, such as field misalignments and asymmetric confining potentials.

\section{ACKNOWLEDGMENTS}

We would like to thank Professor M. Charlton and Mr. A. Deutsch for helping in the editing of the paper. This work has been supported by the National Science Foundation, and U.S. DOE Division of High Energy Physics, Grant No. DEFG02-04ER41289 and was partially supported by the ISF (Israel).

${ }^{1}$ M. Amoretti, C. Amsler, G. Bonomi, A. Bouchta, P. Bowe, C. Carraro, C. L. Cesar, M. Charlton, M. J. T. Collier, M. Doser, ATHENA collaboration et al. Nature (London) 419, 456 (2002).

${ }^{2}$ G. Gabrielse, N. S. Bowden, P. Oxley, A. Speck, C. H. Storry, J. N. Tan, M. Wessels, D. Grzonka, W. Oelert, G. Schepers, ATRAP Collaboration et al., Phys. Rev. Lett. 89, 213401 (2002).

${ }^{3}$ J. S. de Grassie and J. H. Malmberg, Phys. Fluids 23, 63 (1980).

${ }^{4}$ B. I. Deutch, F. M. Jacobsen, L. H. Andersen, P. Hvelplund, H. Knudsen, M. H. Holzcheiter, M. Charlton, and G. Laricchia, Phys. Scr., T 22, 248 (1988).

${ }^{5}$ G. Gabrielse, S. L. Rolston, L. Haarsma, and W. Kells, Phys. Lett. A 129, 38 (1988).

${ }^{6}$ T. Bergeman, G. Erez, and H. Metcalf, Phys. Rev. A 35, 1535 (1987).

${ }^{7}$ E. P. Gilson and J. Fajans, Phys. Rev. Lett. 90, 015001 (2003).

${ }^{8}$ M. Holzscheiter, M. Charlton, and M. M. Nieto, Phys. Rep. 402, 1 (2004).

${ }^{9}$ T. M. Squires, P. Yesley, and G. Gabrielse, Phys. Rev. Lett. 86, 5266 (2001)

${ }^{10}$ J. Fajans, W. Bertsche, K. Burke, S. F. Chapman, and D. P. van der Werf, Phys. Rev. Lett. 95, 155001 (2005)

${ }^{11}$ J. Fajans and A. Schmidt, Nucl. Instrum. Methods Phys. Res. A 521, 318 (2004)

${ }^{12}$ G. Andresen, W. Bertsche, A. Boston, P. D. Bowe, C. L. Cesar, S. Chapman, M. Charlton, M. Chartier, A. Deutsch, J. Fajans, M. C. Fujiwara, R. Funakoshi, D. R. Gill, K. Gomberoff, J. S. Hangst, R. S. Hayano, R. Hydomako, M. J. Jenkins, L. V. Jørgensen, L. Kurchaninov, N. Madsen, P. Nolan, K. Olchanski, A. Olin, A. Povilus, F. Robicheaux, E. Sarid, D. M. Silveira, J. W. Storey, H. H. Telle, R. I. Thompson, D. P. van der Werf, J. S. Wurtele, and Y. Yamazaki, Phys. Rev. Lett. 98, 023402 (2007).

${ }^{13} \mathrm{G}$. Andresen et al., "Antiproton-positron interactions in an antihydrogen trap" (to be submitted).

${ }^{14}$ M. Amoretti, C. Canali, C. Carraro, M. Doser, V. Lagomarsino, G. Manuzio, G. Testera, and S. Zavatarelli, Phys. Lett. A 360, 141 (2006).

${ }^{15}$ D. P. Grote, A. Friedman, I. Haber, and J.-L. Vay, AIP Conf. Proc. 749, 55 (2005).

${ }^{16}$ W. Bertsche, A. Boston, P. D. Bowe, C. L. Cesar, S. Chapman, M. CharIton, M. Chartier, A. Deutsch, J. Dilling, J. Fajans, ALPHA Collaboration et al., AIP Conf. Proc. 796, 301 (2005).

${ }^{17}$ W. Bertsche, A. Boston, P. D. Bowe, C. L. Cesar, S. Chapman, M. CharIton, M. Chartier, A. Deutsch, J. Dilling, J. Fajans, ALPHA Collaboration et al., Nucl. Instrum. Methods Phys. Res. A 566, 746 (2006).

${ }^{18}$ R. H. Cohen, A. Friedman, M. Kireeff Covo, S. M. Lund, A. W. Molvik, F. M. Bieniosek, P. A. Seidl, J.-L. Vay, P. Stoltz, and S. Veitzer, Phys. Plasmas 12, 056708 (2005).

${ }^{19}$ K. Gomberoff, J. Wurtele, A. Friedman, D. Grote, and J.-L. Vay, J. Comput. Phys. 225, 1736 (2007).

${ }^{20} \mathrm{~K}$. Gomberoff, J. Wurtele, J. Fajans, A. Friedman, D. Grote, J.-L. Vay, and R. H. Cohen, Phys. Plasmas 14, 052107 (2007).

${ }^{21}$ W. Bertsche, J. Fajans, and L. Friedland, Phys. Rev. Lett. 91, 265003 (2003).

${ }^{22}$ V. Gorgadze, T. Pasquini, J. Fajans, and J. S. Wurtele, AIP Conf. Proc. 692, 30 (2003).

${ }^{23}$ F. Peinetti, F. Peano, G. Coppa, and J. S. Wurtele, J. Comput. Phys. 218, 102 (2006). 